Luigi A. Pini

Daria Brovia

\section{Different characteristics of triptans}

\author{
L.A. Pini $(\varangle) \cdot$ D. Brovia \\ Centro Cefalee, Università di Modena e \\ Reggio Emilia, \\ Via del Pozzo 71, I-41100 Modena, Italy \\ e-mail: pinila@unimo.it \\ Tel.: +39-50-4224065 \\ Fax: +39-59-4224069
}

\begin{abstract}
Despite the pharmacokinetic differences among triptans and the variety of ways of administration, the clinical differences in everyday use of these drugs do not allow a largely accepted decisional tree. There are a number of comparative trials showing similar results with regard to efficacy, safety, and tolerability of these drugs. This means that the patient's preference is one of the most important decisive factors in choosing one triptan
\end{abstract}

over another. A good migraine therapy requires a balance between patient satisfaction and drug efficacy and safety. All the marked triptans show a good benefit-risk ratio, and comorbidity should be considered when choosing between different triptans.

Key words Triptans •

Pharmacokinetics • Efficacy • Safety • Anti-migraine drugs

\section{Introduction}

The observation that serotonin is involved in migraine led to the development of drugs acting on the serotonergic receptors. Historically, ergot derivatives were the first class of drug acting on a series of receptors, namely, dopaminergic, adrenergic, and serotonergic; triptans were a novel class of drugs acting as selective agonists of 5-HT $1 \mathrm{~B} / 1 \mathrm{D}$ receptors $[1,2]$.

Since the introduction in 1983 of the first triptan, sumatriptan, research has led to the development of the socalled second-generation triptans: zolmitriptan, naratriptan, rizatriptan, almotriptan, eletriptan, and frovatriptan.

One of the most frequently asked question is "Which is the best triptan?" It is difficult to find an answer, because the significance of "best" depends on the features that would serve to discriminate the triptans (i.e., pharmacokinetics, efficacy, potency, safety, rapidity of action, etc.). Moreover, it is difficult to predict the patient's clinical response to a given triptan, and in addition the patient's preference is a complex parameter that depends on unique and individual factors typical to that subject. In conclusion, the answer depends on the point of view that we are taking.

The pharmaceutical formulation remains an important parameter for the evaluation of overall efficacy. Injectable sumatriptan is the most rapidly and potently effective triptan: after $10 \mathrm{~min}$ it provides headache relief (major relief compared with placebo) and by $2 \mathrm{~h}$ headache relief is reported by $80 \%$ of patients; it also acts on migraine-associated symptoms.

Intranasal administration provides significant relief within 40-60 min, and seems to be useful even in the treatment of short headaches such as cluster headache [3-7].

Several oral triptans can provide headache relief within 60-120 min; however, in the case of intense pain that tends to increase quickly in which nausea and vomiting are associated subcutaneous injection or a nasal spray formulation are more appropriate. Almotriptan 
and naratriptan show a lower incidence of side effects, in particular chest and central nervous system symptoms [8].

\section{Pharmacokinetics of triptans}

Table 1 presents the pharmacokinetic parameters of triptans. Sumatriptan shows a low oral bioavailability (14\%), a short elimination half-life, and a modest time to maximal serum concentration. This is the basis for the development of drugs with different pharmacokinetic and interaction profiles.

Zolmitriptan, naratriptan, rizatriptan, and frovatriptan show gender differences in their pharmacokinetics, whereas with almotriptan there are no differences between men and women, but the pharmacokinetic profile is affected by age. These differences are not considered clinically relevant, but in patients with severe hepatic impairment, a reduction of the dose may be necessary, in particular when using zolmitriptan.

Almotriptan plasma concentration levels are increased by $37 \%$ by co-administration of moclobemide and vera- pamil. Sumatriptan does not interact with propranolol, alcohol, or paroxetine, but plasma levels are significantly increased by moclobemide.

The half-life of zolmitriptan is prolonged by $32 \%$ when co-administered with propanolol; moreover, moclobemide increases the area under the curve (AUC) and the active metabolites of this triptan.

Plasma concentration levels of rizatriptan are increased by $70 \%$ by propranolol, and it is therefore advisable to maintain the lower dose of $5 \mathrm{mg}$. Moclobemide augments rizatriptan plasma concentration, and the co-administration with any monoamine oxidase type A inhibitor is contraindicated. The metabolic interactions of triptans are summarized in Table 2 [9].

\section{Efficacy}

Pharmacokinetic differences among the new triptans are not related to clinical efficacy. Table 3 reports the main parameters used to evaluate the efficacy and safety of triptans.

The oral triptans share the same ability to provide pain relief within 30-60 min. Concerning the reduction of

Table 1 Pharmacokinetic profiles of triptans (AUC, area under the curve)

\begin{tabular}{|c|c|c|c|c|c|}
\hline Triptan & $T_{\max }(\mathrm{h})$ & Oral bioavailability (\%) & $T_{1 / 2}(\mathrm{~h})$ & AUC (mg/l h) & Active metabolites \\
\hline Sumatriptan $50 \mathrm{mg}$ & 2 & 14 & 2 & 118 & No \\
\hline Sumatriptan $100 \mathrm{mg}$ & 1.5 & 14 & 2 & 158 & No \\
\hline Rizatriptan $5 \mathrm{mg}$ & 1.2 & 38 & 1.4 & 17.4 & Yes \\
\hline Rizatriptan $10 \mathrm{mg}$ & 1 & 40 & 2 & 50 & Yes \\
\hline Zolmitriptan $2.5 \mathrm{mg}$ & 2 & 46 & 2.6 & 17 & Yes \\
\hline Naratriptan $2.5 \mathrm{mg}$ & 2 & 74 & 5.5 & 98 & No \\
\hline Naratriptan $5 \mathrm{mg}$ & 2 & 68 & 5.3 & 200 & No \\
\hline Naratriptan $10 \mathrm{mg}$ & 1.5 & 68 & 5.5 & 387 & No \\
\hline Eletriptan $40 \mathrm{mg}$ & 1.8 & 50 & 5.3 & 670 & Yes \\
\hline Eletriptan $80 \mathrm{mg}$ & 1.4 & 50 & 6.3 & 1,661 & Yes \\
\hline Frovatriptan $2.5 \mathrm{mg}$ & 3 & 29.6 & 25.7 & 94 & No \\
\hline Frovatriptan $40 \mathrm{mg}$ & 5 & 17.5 & 29.7 & 881 & No \\
\hline Almotriptan $12.5 \mathrm{mg}$ & 2.5 & 49.5 & 3.1 & 80 & No \\
\hline Almotriptan $25 \mathrm{mg}$ & 1.5 & 69 & 3.19 & 558.5 & No \\
\hline
\end{tabular}

Table 2 Metabolic interactions of triptans

\begin{tabular}{lcccccrr}
\hline & Almotriptan & Eletriptan & Frovatriptan & Naratriptan & Rizatriptan & Sumatriptan & Zolmitriptan \\
\hline Renal & No & Yes & No & No & Yes & No & No \\
Hepatic & No & No & No & No & Yes & Yes & Yes \\
MAOI & No & No & No & No & Yes & Yes & Yes \\
SSRI & No & No & No & No & No & Yes & No \\
CYP3A4 inhibitors & No & Yes & No & No & No & Yes & No \\
Active metabolites & No & Yes & No & No & Yes & No & Yes \\
Propranolol & No & No & No & No & Yes & No & No \\
\hline
\end{tabular}


Table 3 Clinical efficacy and safety parameters of triptans

\begin{tabular}{ll}
\hline Efficacy parameters & Safety parameters \\
\hline $\begin{array}{l}\text { Therapeutic gain }=\text { response rate in treated subjects/response rate } \\
\text { in control group }\end{array}$ & $\begin{array}{l}\text { Absolute risk increase = adverse effects (AEs) rate in treated } \\
\text { subjects/AEs rate in control group } \\
\text { Relative benefit increase }=\text { therapeutic gain/response rate }\end{array}$ \\
$\begin{array}{l}\text { Relative risk increase }=\text { absolute risk increase/AEs rate in control } \\
\text { group }\end{array}$ \\
Number needed to treat $=100 /$ therapeutic gain $(\mathrm{NNT})$ & Number needed to harm $=100 /$ absolute risk increase $(\mathrm{NNH})$ \\
\hline
\end{tabular}

headache recurrence, the rates are similar for rizatriptan, zolmitriptan, and sumatriptan; however, with naratriptan the rates of recurrence were low in some studies versus placebo, and significantly lower in comparison with sumatriptan in a double-blind crossover study involving patients with a high percentage of recurrence.

Frovatriptan has the longest half-life and shows a lower rate of headache recurrence.

The intrapatient consistency (percentage of crisis treated successfully in an individual patient) rather than the population consistency (percentage of patients responding to consecutive attacks) is the most appropriate method of evaluating the consistency of a drug.

Studies with oral sumatriptan show that $64 \%$ of patients achieve pain relief in at least two of three attacks and $33 \%$ had pain relief in all three crises.

Almotriptan, the triptan with the highest bioavailability $(70 \%)$, provided pain relief in at least two attacks in $75 \%$ of patients treated with $12.5 \mathrm{mg}$, and almost $50 \%$ of these patients were pain-free in all three attacks. These data suggest a possible relationship between oral availability and consistency of response. On the other hand, intrapatient response consistency with rizatriptan (bioavailability of $40 \%$ ) was higher than for almotriptan: pain relief in $86 \%$ at $2 \mathrm{~h}$ for two of three attacks [9].

\section{Safety}

The most frequently reported adverse events are dizziness/vertigo, somnolence, nausea/vomiting, asthenia, dry mouth, chest symptoms, and paresthesias. The tolerability profiles of rizatriptan, sumatriptan, zolmitriptan, and eletriptan are similar.

The incidence of new adverse events is lower with naratriptan, frovatriptan, and almotriptan: the tolerability profile of almotriptan $12.5 \mathrm{mg}$ is similar to placebo.

Almotriptan and naratriptan show a low incidence of chest symptoms: $0.1 \%-0.2 \%$ and $1 \%$, respectively.

Although the potential for any triptan to cause constriction of the coronary arteries is very low at a therapeutic dosage, these drugs are contraindicated in patients who have coronary artery disease [10-12].

\section{References}

1. Hargraves RJ, Shepherd SL (1999) Pathophysiology of migraine - new insights. Can J Neurol Sci 26[Suppl 3]:S12-S19

2. Tfelt-Hansen P, Saxena PR, Daholf C et al (2000) Ergotamine in the acute treatment of migraine: a review and European consensus. Brain 123:9-18

3. Cady RK, Wendt JK, Kirchner JR, Sargent JD et al (1991) Treatment of acute migraine with subcutaneous Sumatriptan. JAMA 265:2831-2835

4. The Subcutaneous Sumatriptan International Study Group (1991) Treatment of migraine attacks with Sumatriptan. N Engl J Med 325:316-321

5. Daholf C (1999) Sumatriptan nasal spray in the acute treatment of migraine: a review of clinical studies. Cephalalgia 19:769-778
6. Pfaffenrath V, Cunin G, Sjionell G, Prendergast S (1998) Efficacy and safety of Sumatriptan tablets $(25 \mathrm{mg}$, $50 \mathrm{mg}$ and $100 \mathrm{mg}$ ) in the acute treatment of migraine: defining the optimum doses of oral Sumatriptan. Headache 38:184-190

7. Sargent J, Kirchner JR, Davis R, Kirkhart B (1995) Oral sumatriptan is effective and well tolerated for the acute treatment of migraine: evaluation of three dosage strengths. Neurology 45[Suppl 7]:S10-S14

8. Robert M, Cabarrocas X, Fernandez FJ, Zayas JM, Ferrer P, for and on behalf of the Almotriptan Multiple Attacks Study Group (1998) Efficacy and tolerability of oral Almotriptan in the treatment of migraine. Cephalalgia 18:406
9. Dahlof CG, Dodick DW, Dowson AJ, Pascual J (2002) How does Almotriptan compare to other triptans? A review of data from placebo-controlled clinical trials. Headache 42:99-113

10. Visser WH, Jaspers NM, de Vriend RH, Ferrari MD (1996) Risk factors for headache recurrence after Sumatriptan: a study of 366 migraine patients. Cephalalgia 16:264-269

11. Ferrari MD (1999) How to assess and compare drugs in the management of migraine: success rates in terms of response and recurrence. Cephalalgia 19[Suppl 23]:2-8

12. Maassen Van Den Brink A, Reekers M, Baxx WA, Ferrari MD, Saxena PR (1998) Coronary side-effect potential of current and prospective antimigraine drugs. Circulation 98:25-30 